\title{
Chronic thromboemboli pulmonary hypertension in patient with Eisenmenger syndrome and large patent ductus arterius
}

\author{
Ke T Tran, Le T Pham* and Luan H Quang \\ General Hospital of Gia Lai Province, 132 Tôn Thất Tùng, Phù Đổng, Thành phố Pleiku, Gia Lai 600000, Vietnam
}

\begin{abstract}
Chronic thromboemboli pulmonary hypertension (CTEPH) is a group 4 of pulmonary hypertensions, related to clot blocking in the pulmonary arteries in lungs. Patients with CTEPH have varies typical or atypical symptoms, which are not specific and the diagnosis of CTEPH is a challange to clinicians. Chronic thromboembolic pulmonary hypertension (CTEPH) most often results from obstruction of the pulmonary vascular bed by non-resolving thromboemboli. Chronic thromboembolic pulmonary hypertension can arise in patients after acute or recurrent pulmonary emboli or deep venous thrombosis. The incidence of CTEPH is not known, but recent studies suggest that $1 \%$ to $3.8 \%$ of patients develop the condition within 2 years of acute pulmonary embolism. 64 -row CT of the pulmonary arteries can yield diagnostically excellent image quality and can delineate the typical angiographic findings in CTEPH such as complete obstruction, bands and webs and intimal irregularities as accurate and reliable as DSA. With additional thick MIPs it is possible to get an instant overview of the entire pulmonary arterial tree, which helps to demonstrate the pathology related of CTEPH similar to DSA. When the diagnosis of CTEPH is confirmed, anticoagulant should be used. Pulmonary thromboendarectomy is the most optimal therapeutic beside Pulmonary artery Balloon dilation. We found a rare case diagnosed CTEPH with Eisenmenger syndrome, large PDA by CT scanner and Echocardiography.
\end{abstract}

\section{Case presentation}

A 51-year-old female admitted for dyspnea, cyanotic, decrease of excirse capacity, cough, limb edema and right chest pain for several days. The patient was diagnosed with heart failure NYHA III, hypertension, pulmonary hypertension 1 year ago and treated with ARB, diuretic, sildenafil but she had interrupted medicines for 2 months because of financial issue. In the Emergency Room, her vital signs: BP 140/100 mmHg, HR :130 bpm, Temperature 37.8 Celsius degree, Respirator rate: $30 \mathrm{bpm}$.

Lung: crackle in bilateral fields, SPO2: 50\%, Heart sound: murmur $4 / 6$ in apex, clubbing fingers (Figure 1).

ECG: Sinus rhythm with ventricular rate almost 92bpm, Right axis deviation, RBBB, pulmonary $\mathrm{P}$ wave (Figure 2).

Echocardiography: EF 40\%, right ventricular dilation (RVDD:40mm) D shape RV, Right ventricular hypertrophy, IVC: $30 \mathrm{~mm}$, severe Tricuspid valve regurgitation, PAPs: $100 \mathrm{mmHg}$, dilated Pulmonary artery trunk and right pulmonary artery. Mc'cornell sign, severe Pulmonary valve insufficient, Severe pulmonary hypertension, no left to right or right to left shunt was detective (Figures 3 and 4).

Thoracic Xray when admitted ER: Pulmonary trunk dilation, cardiomegaly, consolidation in inferior lobe right lung (Figure 5).

Thoracic Xray after 7 days: progressive Hampton sign appears (Figure 6).

Thoracic MSCT: dilated Right heart, consolidation in inferior lobe of right lung, massive thrombus in distal right pulmonary artery branch, Polo mint sign in right pulmonary artery branch, large PDA (Figure 7). The patient was treated with ARB, Diuretic, anticoagulant, CPAP,
Antibiotic. After 14 days, the patient was more stable and discharged with oral anticoagulant, ARB, diuretic [1-3].

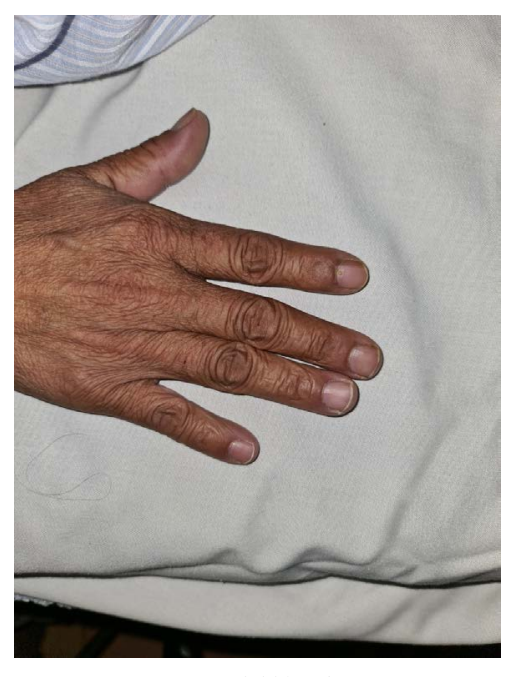

Figure 1. Clubbing fingers

*Correspondence to: Le Tra Pham, General Hospital of Gia Lai Province, 132 Tôn Thất Tùng, Phù Đổng, Thành phố Pleiku, Gia Lai 600000, Vietnam, E-mail: leanhkado@gmail.com

Received: January 16, 2021; Accepted: January 22, 2021; Published: February 02,2021 


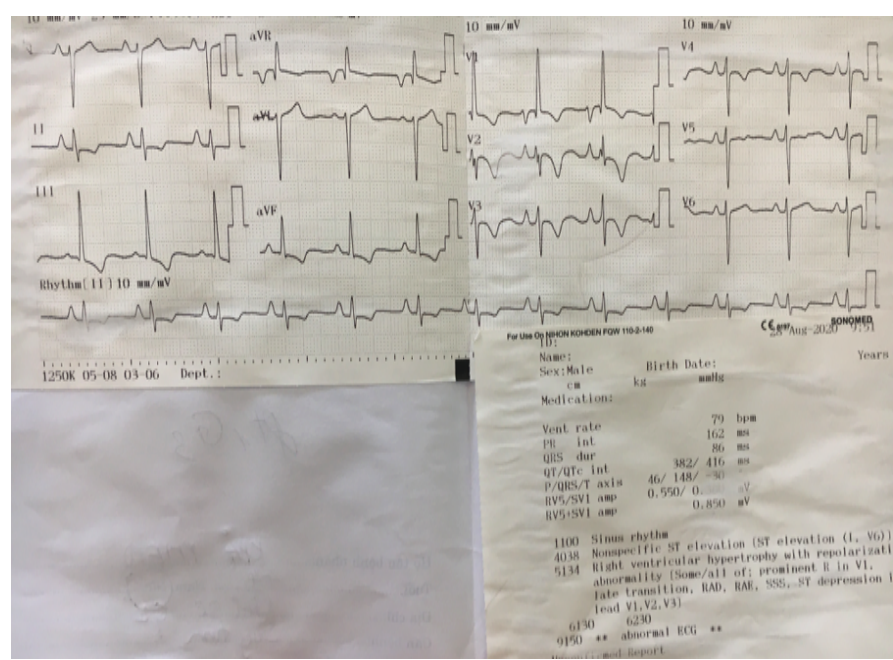

Figure 2. Sinus rhythm with ventricular rate almost $92 \mathrm{bpm}$, Right axis deviation, RBBB pulmonary $\mathrm{P}$ wave

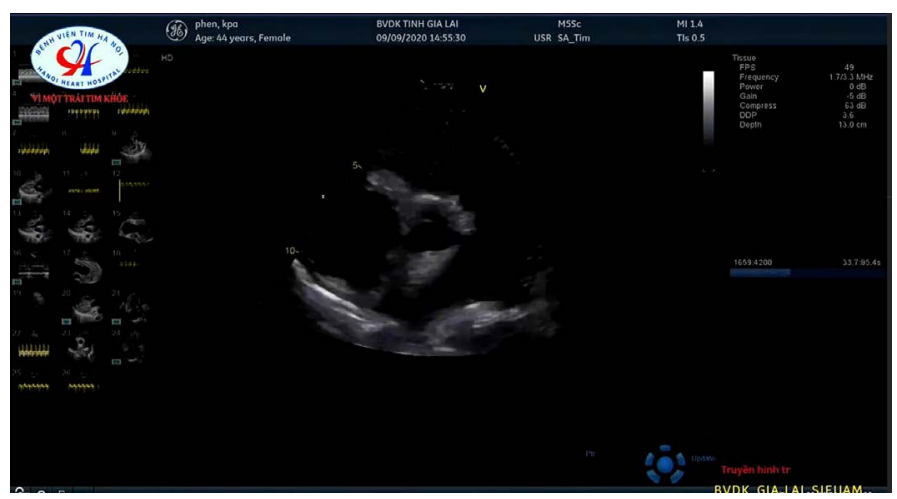

Figure 3. Dilated Pulmonary artery trunk and right pulmonary artery

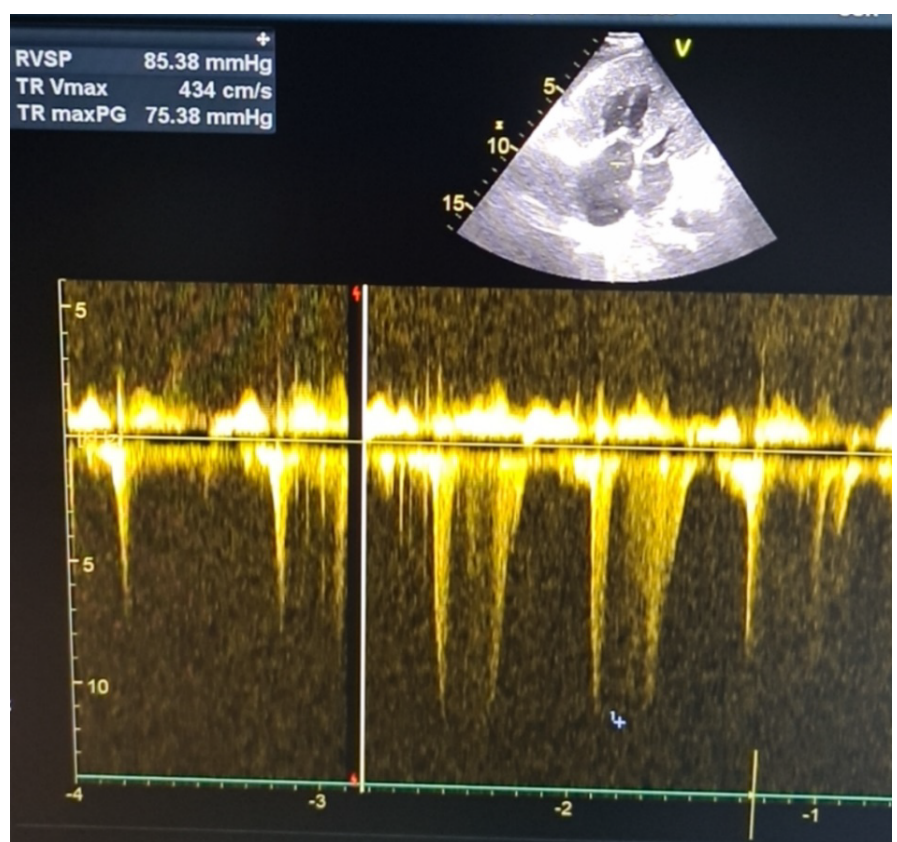

Figure 4a. Severe Tricuspid valve regurgitation, severe pulmonary hypertension

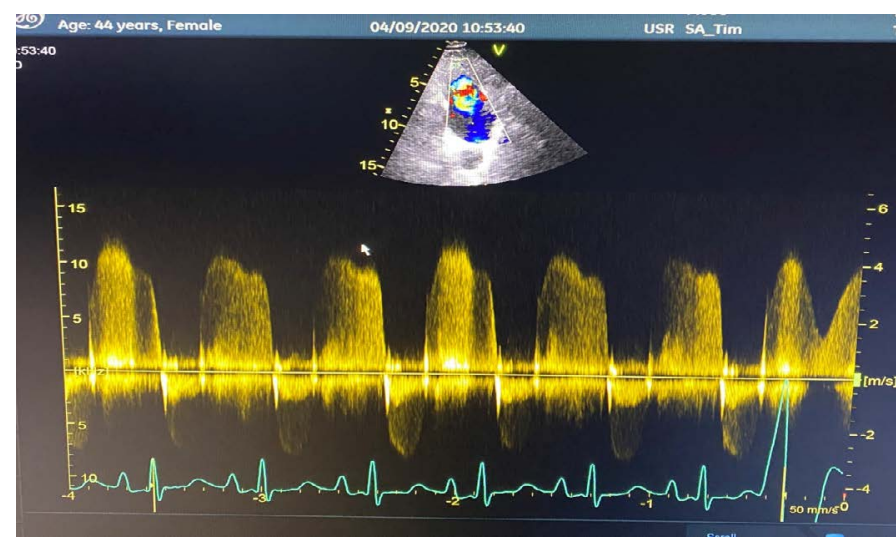

Figure 4b. Severe pulmonary valve insufficient, severe pulmonary hypertension

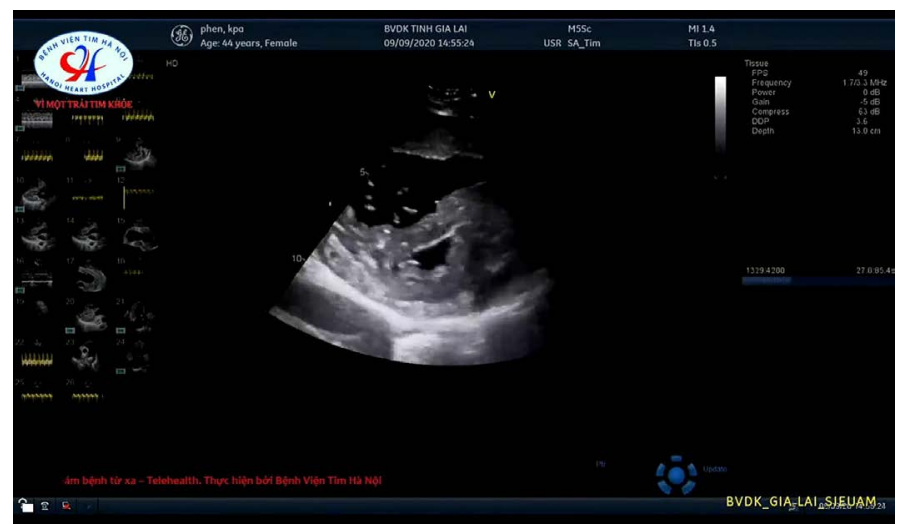

Figure 4c. Right ventricular dilation (RVDD: 40mm) D shape RV, Right ventricular hypertrophy

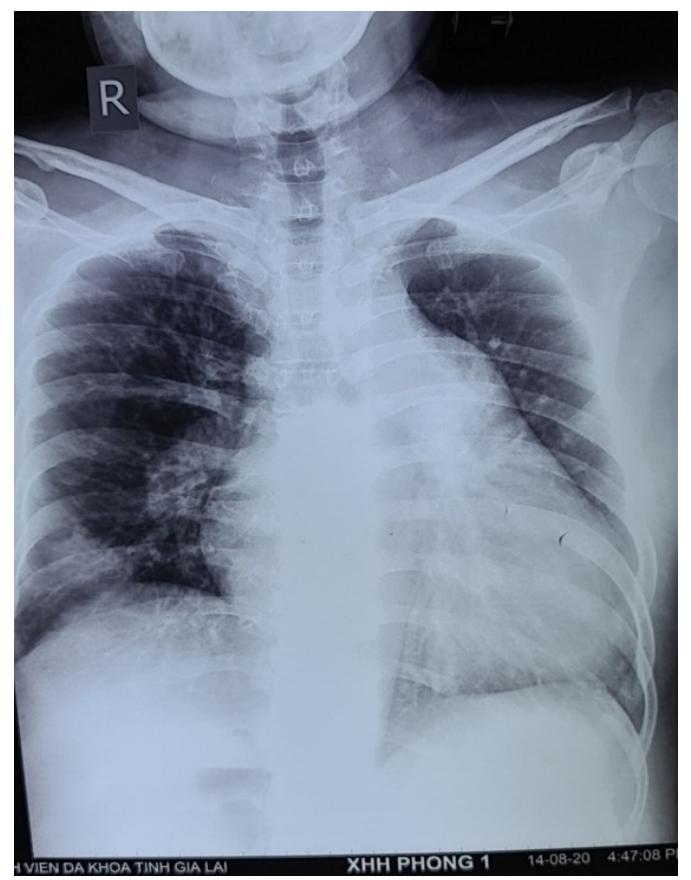

Figure 5. Pulmonary trunk dilation, cardiomegaly, consolidation in inferior lobe right lung 


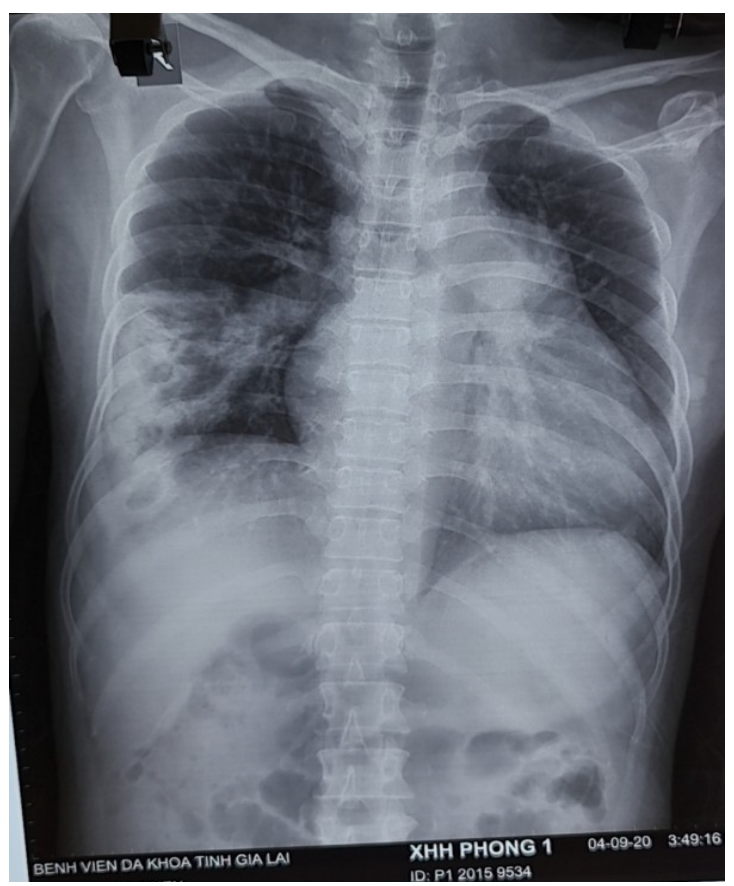

Figure 6. Progressive Hampton sign appears

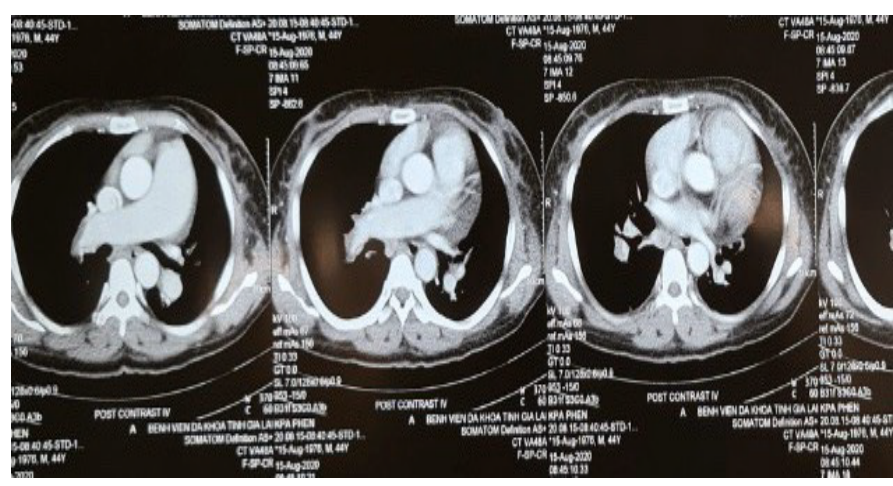

Figure 7a. Dilated Right heart, dilate right pulmonary artery, massive thrombus in dista right pulmonary artery branch, Polo mint sign in right pulmonary artery branch

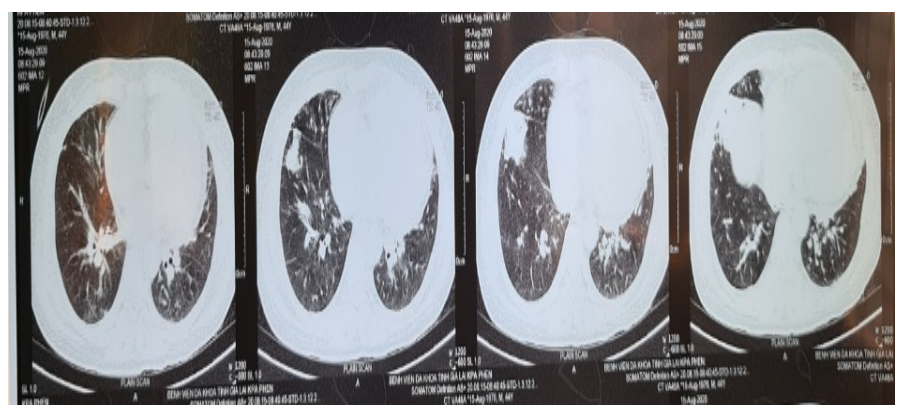

Figure 7b. Consolidation in inferior lobe of right lung

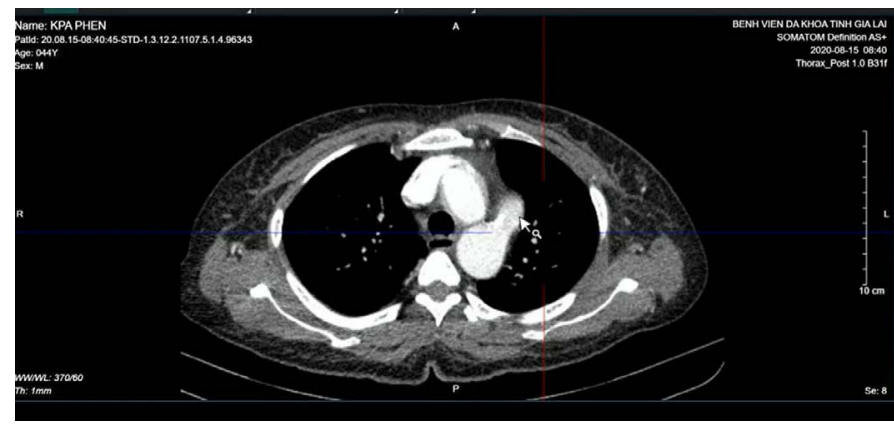

Figure 7c. Large PDA in axial plane (white arrow)

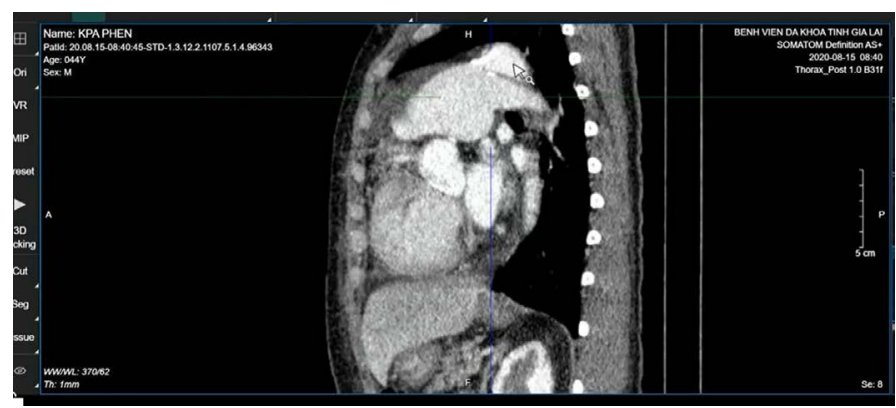

Figure 7d. Large PDA in sagittal plane (white arrow)

\section{References}

1. Becattini C, Agnelli G, Pesavento R, Silingardi M, Poggio R, et al. (2006) Incidence of chronic thromboembolic pulmonary hypertension after a first episode of pulmonary embolism. Chest 130: 172-175. [Crossref]

2. Pepke-Zaba J, Delcroix M, Lang I, Mayer E, Jansa P, et al. (2011) Chronic thromboembolic pulmonary hypertension (CTEPH): results from an international prospective registry. Circulation 124: 1973-1981. [Crossref]

3. Reichelt A, Hoeper MM, Galanski M, Keberle M (2008) Chronic thromboembolic pulmonary hypertension: Evaluation with 64-detector row CT versus digital substraction angiography. Eur J Radiol 71: 49-54. [Crossref]

Copyright: (C2021 Tran KT. This is an open-access article distributed under the terms of the Creative Commons Attribution License, which permits unrestricted use, distribution, and reproduction in any medium, provided the original author and source are credited. 